\title{
Eta and Etaprime Photoproduction on the Nucleon with the Isobar Model EtaMAID2018
}

L. Tiator ${ }^{1, *}$, M. Gorchtein ${ }^{1}$, V. Kashevarov ${ }^{1}$, K. Nikonov ${ }^{1}$, M. Ostrick ${ }^{1}$, M. Hadžimehmedović ${ }^{2}$, R. Omerović ${ }^{2}$, H. Osmanović ${ }^{2}$, J. Stahov ${ }^{2}$, and A. Švarc ${ }^{3}$

${ }^{1}$ Institut für Kernphysik, Johannes Gutenberg-Universität Mainz, Mainz, Germany

${ }^{2}$ University of Tuzla, Faculty of Natural Sciences and Mathematics, Univerzitetska 4, 75000 Tuzla, Bosnia and Herzegovina

${ }^{3}$ Rudjer Bošković Institute, Bijenička cesta 54, P.O. Box 180, 10002 Zagreb, Croatia

\begin{abstract}
The isobar model EtaMAID has been updated with new and high precision data for $\eta$ and $\eta^{\prime}$ photoproduction on protons and neutrons from MAMI, ELSA, GRAAL and CLAS. The background is described in a recently developed Regge-cut model, and for the resonance part the whole list of nucleon resonances has been investigated with $21 N^{*}$ states contributing to $\eta$ photoproduction and $12 N^{*}$ states contributing to $\eta^{\prime}$ photoproduction. Finally, the possibility of a narrow resonance near $W=1900 \mathrm{MeV}$ is discussed, that would be able to explain unexpected energy and angular dependence of observables in $p\left(\gamma, \eta^{\prime}\right) p$ near $\eta^{\prime}$ threshold.
\end{abstract}

\section{Introduction}

EtaMAID is an isobar model to describe eta and etaprime photoproduction on the nucleon. It is based on $s$ - and $u$-channel Born terms, $t$-channel vector meson exchanges and $s$-channel nucleon resonance excitations ( $\Delta$ resonances are forbidden by isospin).

As a part of the Mainz MAID project [1, 2], EtaMAID was introduced in 2001 [3] as a model with eight prominent nucleon resonances: $N(1535) \frac{1}{2}^{-}\left(S_{11}\right), N(1650) \frac{1}{2}^{-}\left(S_{11}\right)$, $N(1710) \frac{1}{2}^{+}\left(P_{11}\right), N(1720) \frac{3}{2}^{+}\left(P_{13}\right), N(1520) \frac{3}{2}^{-}\left(D_{13}\right), N(1700) \frac{3}{2}^{-}\left(D_{13}\right), N(1675) \frac{5}{2}^{-}\left(D_{15}\right)$, and $N(1680) \frac{5}{2}^{+}\left(F_{15}\right)$. The background was modeled with Born terms and $t$-channel vector meson exchanges of $\omega$ and $\rho$ mesons. The model was developed for photo- and electroproduction on protons and neutrons and was well fitted to the few available data in 2001.

In 2003 an update was performed for eta and etaprime photoproduction on the proton [4], where newly measured data were fitted and a Regge background was introduced for a better description of high-energy data.

Since that time a lot of developments occurred, first of all for the experimental data base. There was a huge effort at several accelerator facilities to combine high intensity polarized photon beams with modern $4 \pi$ detectors and spin-polarized targets. The entire dataset used in our new analysis was obtained at Mainz, Bonn, JLab and GRAAL between 2007-2017, see table III of Ref. [5].

Starting in 2015, a new major update of EtaMAID was undertaken with various new aspects. First we extended the energy range and took into consideration all reported nucleon

*e-mail: tiator@uni-mainz.de 
resonances [6], where only few of them showed no significance in respect to the data fitting. Second we modified the background and investigated different Regge approaches in order to describe the high-energy data, including polarization observables. Third we analyzed all four channels $\eta p, \eta n, \eta^{\prime} p$, and $\eta^{\prime} n$ simultaneously as all nucleon resonances can contribute to two or even all four channels with the same mass and width. A detailed write-up of the EtaMAID2018 model with many figures and tables can be found in Ref. [5].

\section{The isobar model}

In the isobar model the photoproduction amplitudes of $\eta$ and $\eta^{\prime}$ mesons are written in terms of nucleon resonance excitations in generalized Breit-Wigner forms and in non-resonant background amplitudes.

For a specific partial wave $\alpha=\alpha(\ell, j=\ell \pm 1 / 2, \mathcal{M})$, where $\ell$ is the angular momentum of the $\eta N$ system in the final state, $j$ is the total spin and $\mathcal{M}$ stands either for an electric (E) or magnetic (M) transition. The total partial wave amplitude can be written as a sum of a background amplitude $t^{\alpha, b}$ and a resonance amplitude $t^{\alpha, r}$

$$
t_{\gamma, \eta}^{\alpha}(W)=t_{\gamma, \eta}^{\alpha, b}(W)+t_{\gamma, \eta}^{\alpha, r}(W)
$$

In photoproduction we identify the partial wave amplitudes directly with the electromagnetic multipoles $E_{\ell \pm}$ and $M_{\ell \pm}$.

Traditionally, the background amplitude is taken as a sum of Born terms and $t$-channel meson exchange contributions

$$
t_{\gamma, \eta}^{\alpha, b}(W)=t_{\gamma, \eta}^{\alpha, B o r n}(W)+t_{\gamma, \eta}^{\alpha, V M}(W)
$$

The Born terms for $\eta$ and $\eta^{\prime}$ photoproduction play a minor role due to the small coupling constants. For the $t$-channel exchanges we use Regge amplitudes including Regge trajectories and Regge cuts. In our recent work on Regge phenomenology [7] this model has already been fitted to the high-energy world data of eta photoproduction. An application of this approach in EtaMAID for the resonance region now raises the problem of double-counting due to the quark-hadron duality issue: The sum of an infinite series of $s$-channel nucleon resonances is equivalent to an infinite sum of $t$-channel meson resonances. In the EtaMAID model, we do have an infinite sum of $t$-channel exchanges in our Regge amplitudes, but the sum of $s$-channel resonances remains finite. Nevertheless some double counting occurs and in our new approach we approximately remove the double counting by multiplication of a damping factor to the Regge amplitudes. This damping factor keeps the well described high-energy regime, but damps out the Regge amplitudes in the resonance region down to threshold,

$$
\begin{aligned}
t & =\sum_{i=1}^{\infty} t_{s}^{\text {Res }}{ }^{i}=\sum_{i=1}^{\infty} t_{t}^{\text {Res }} \\
& =\sum_{i=1}^{N} t_{s}^{\text {Res }}+\left[\sum_{i=1}^{\infty} t_{t}^{\text {Res }}{ }_{i}-\sum_{i=1}^{N} t_{s}^{\text {Res }}\right] \\
& \approx \sum_{i=1}^{N} t_{s}^{\text {Res }}{ }^{i}+F_{d}(W) t^{\text {Regge }}
\end{aligned}
$$

with

$$
F_{d}(W)=\left(1-e^{-\frac{W-W_{t h r}}{\Lambda_{R}}}\right) \theta\left(W-W_{t h r}\right)
$$


The cut-off parameter $\Lambda_{R}=974$ (440) $\mathrm{MeV}$ was found in the fit to the data and leads to $F_{d}=0.92(0.99)$ at $W=4 \mathrm{GeV}$ for $\gamma, \eta\left(\gamma, \eta^{\prime}\right)$. At $\eta$ threshold $F_{d}=0$.

The resonance amplitudes are constructed for a given partial wave $\alpha$, as a set of $N_{\alpha}$ nucleon resonances, added as generalized Breit-Wigner (BW) functions with a unitary phase $\phi$ for each resonance,

$$
t_{\gamma, \eta}^{\alpha, r}(W)=\sum_{j=1}^{N_{\alpha}} t_{\gamma, \eta}^{\alpha, B W, j}(W) e^{i \phi_{j}}
$$

We took all listed $N^{*}$ resonances from the Particle Data Tables [6] into consideration and allowed up to seven decay channels: $\pi N, \pi \pi N, \eta N, K \Lambda, K \Sigma, \omega N$ and $\eta^{\prime} N$. The newly introduced unitarity phases turned out to be an important issue for a better description of the data and in particular also for an application of fixed- $t$ dispersion relations [8].

\section{Results}

Our fit uses a large database with angular distributions for cross sections and polarization observables for all four channels, by far most are from the $\eta p$ channel, where even five observables are available, whereas for the $\eta^{\prime} n$ channel only the differential cross sections have been measured. Further details can be found in table III of Ref. [5]. The fit results for the total cross sections, which have not been used in the fit, are presented in Fig. 1 together with corresponding experimental data.
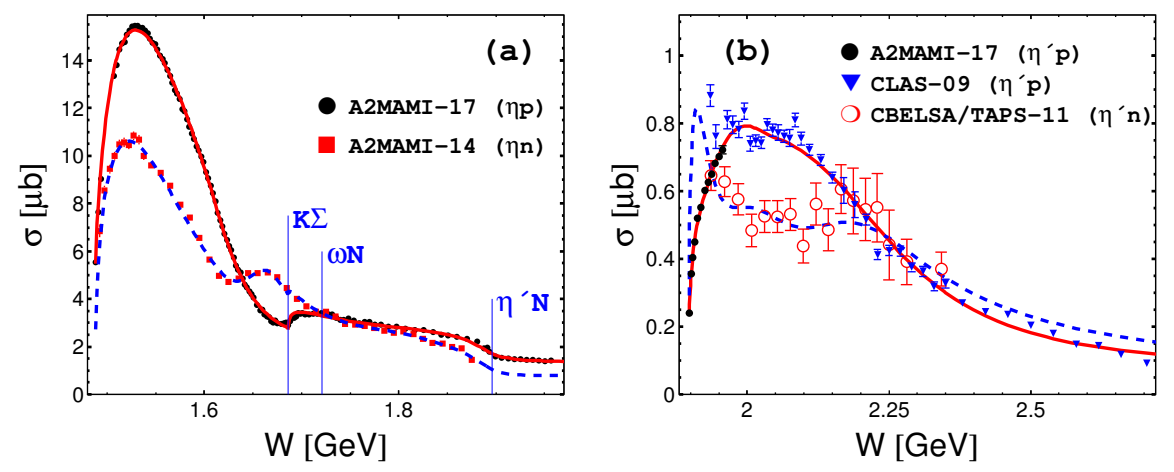

Figure 1. Total cross section for $(\gamma, \eta)$ (a) and $\left(\gamma, \eta^{\prime}\right)$ (b) on protons and neutrons. The solid red and dashed blue lines show our EtaMAID solution for proton and neutron, respectively. The data are from references [9] for $\eta p,[10]$ for $\eta n,[9,11]$ for $\eta^{\prime} p$ and [12] for $\eta^{\prime} n$.

In Fig. 1 (a), there are very interesting features visible at energies $W \approx 1680 \mathrm{MeV}$ and $W \approx 1890 \mathrm{MeV}$, which can be explained by cusp effects due to the opening of new strong channels in the $S$-wave. The cusp in the $\eta p$ total cross section, in connection with the steep rise of the $\eta^{\prime} p$ from its threshold, Fig. 1 (b), is explained by a strong coupling of the $S_{11}(1895)$ resonance to both channels. Unfortunately, there are no data for the $\eta^{\prime} n$ channel near threshold and only one data point exists in the cusp region for the $\eta n$ channel, Fig. 1 (a). Nevertheless our solution demonstrates also a strong coupling of the $S_{11}(1895)$ for the neutron channel.

Other interesting structures are observed as a dip in $\gamma p \rightarrow \eta p$ and a bump in $\gamma n \rightarrow \eta n$ around $W \approx 1680 \mathrm{MeV}$, Fig. 1 (a). Both structures were observed experimentally many times and its existence is unambiguous. However its nature is not yet fully understood. See for 
more details Ref. [13]. Our analysis shows that the narrow bump in $\eta n$ and the dip in $\eta p$ channels have different origin. The first is a result of an interference of few resonances with a dominant contribution of the $P_{11}(1710)$. The second one is mainly a sum of $S_{11}(1535)$ and $S_{11}(1650)$ with opposite signs. However the narrowness of this structure is explained by a cusp effect due to the opening of the $K \Sigma$ decay channel of the $S_{11}(1650)$ resonance.

\section{Partial wave amplitudes}

Compared to pion photoproduction, a comparison of partial waves from different PWA is not straightforward in $\eta$ or $\eta^{\prime}$ photoproduction. First of all, different conventions for isospin matrix elements are used in the literature, which appear as +1 in the $\mathrm{BnGa}$, JüBo and KSU analysis and -1 in the MAID and SAID analysis. Second, for $\eta$ and $\eta^{\prime}$ photoproduction no such convenient unitarity constraints as the Watson Theorem exist, that determine the phases in the low-energy regime. The only, somewhat weaker constraints arise from channel couplings, which are more advanced in coupled-channels approaches as BnGa, JüBo and KSU. In EtaMAID we introduce coupling to other channels only via the Breit-Wigner ansatz and the parametrization of the energy dependent widths. E.g. the $N(1535) \frac{1}{2}^{-}$provides a very strong constraint because of its large branchings of about $50 \%$ for $\pi N$ and $40 \%$ for $\eta N$. For other partial waves, such BW constraints are much less effective.
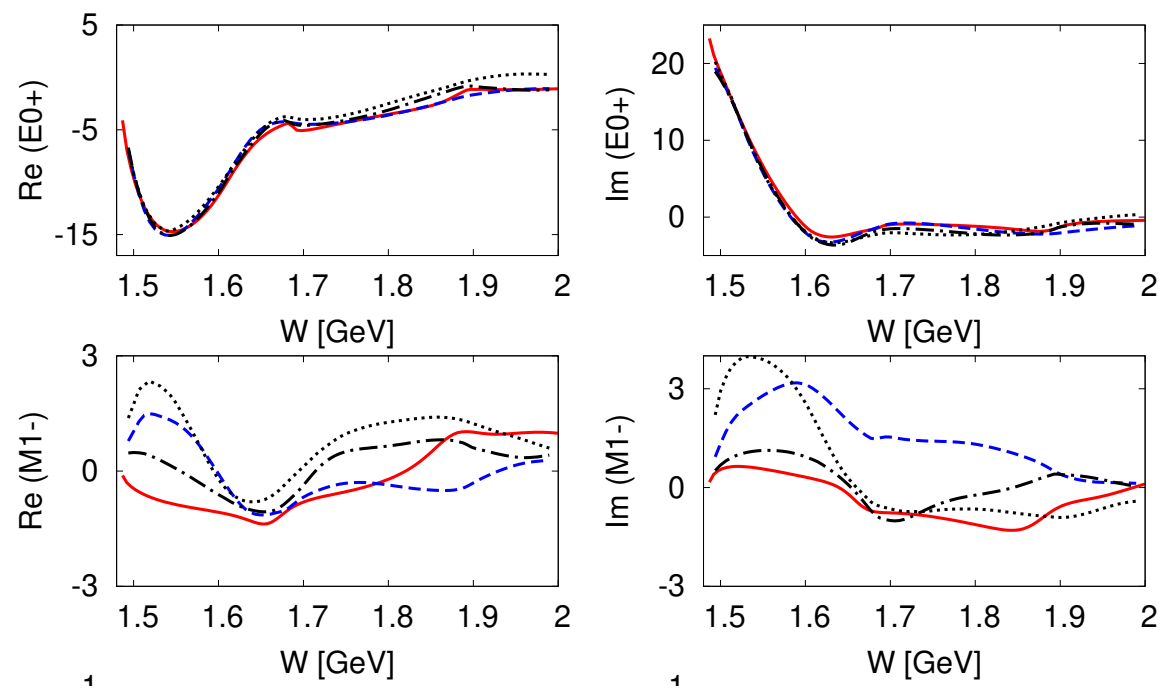

Figure 2. Comparison of $E_{0+}\left(S_{11}\right)$ and $M_{1-}\left(P_{11}\right)$ multipoles for $\gamma p \rightarrow \eta p$, obtained from rotated helicity amplitudes (see Eq. (6)) of different PWA. The solid red lines show our EtaMAID2018 solution. Results of other PWA analyses are shown by the black dash-dotted (BnGa [18]), the black dotted (JüBo [19]), and the blue dashed (KSU [20]) lines. The multipoles are given in units of $\mathrm{mfm}$.

Therefore, even if complete experiments were performed, final ambiguities would remain, which could not be resolved by experimental observables. All physical observables are sums of bi-linear products of amplitudes and conjugated amplitudes, e.g. $\operatorname{Re}\left\{H_{i}(W, \theta) H_{j}^{*}(W, \theta)\right\}$, and are therefore invariant under an overall energy- and angle-dependent phase $\phi(W, \theta)$. This phase depends very much on the models and on couplings with other channels, which finally will always be incomplete. 
For a better comparison between the different newly updated 2018 PWA, that all use practically the same database, we have performed a phase rotation of all amplitudes to our EtaMAID2018 phase,

$$
H_{i}^{B G} \rightarrow \tilde{H}_{i}^{B G}=H_{i}^{B G} \cdot e^{i\left(\phi_{H 1}^{M D}(W, \theta)-\phi_{H 1}^{B G}(W, \theta)\right)}, i=1, \ldots, 4,
$$

where MD stands for the EtaMAID model and BG for any other PWA, as BnGa, JüBo, and KSU. For a detailed discussion of angle-dependent phase ambiguities, see Ref. [14, 15].

In Fig. 2 we compare the multipoles from rotated helicity amplitudes of EtaMAID [5], BnGa [18], JüBo [19], and KSU [20]. While the $S$ wave is practically identical among all solutions, all other partial waves show deviations from small up to huge. Moderate deviations are obeyed in $E_{1+}, M_{1+}, E_{2_{-}}$, and $M_{3_{-}}$, those we can already expect from different fits to the measured data. Other partial waves as $M_{1-}$ (shown in Fig. 2) and $E_{2+}$ show very large deviations, which are most likely due to the incompleteness of the database, where such ambiguities must be expected.

A possible solution of this problem could be obtained along the lines of Ref. [16] by using constraints from fixed- $t$ analyticity. But in addition also improvements of the database with further observables and higher statistics would be very helpful.

\section{Possible narrow resonance $\mathrm{N}(1900)$}

Finally we want to discuss a very new attempt to study possible consequences from a narrow $N(1900)$ state, a few MeV above $\eta^{\prime}$ threshold. Recently, Anisovich et al. [17] have shown that a narrow $N(1900) \frac{3}{2}^{-} D_{13}$ resonance with a mass $M_{R}=1900 \pm 1 \mathrm{MeV}$ and a total width of less than $3 \mathrm{MeV}$ can explain the unexpected energy and angular dependence of the differential cross section $d \sigma / d \Omega$ from A2MAMI and of the beam asymmetry $\Sigma$ from GRAAL. In our EtaMAID analysis we can confirm the possibility for an explanation with a narrow resonance, however, in EtaMAID we would obtain a narrow $S_{11}$ resonance with quantum numbers $\frac{1}{2}^{-}$, mass $M_{R}=1902.6 \pm 1.0 \mathrm{MeV}$ and width $\Gamma_{R}=2.1 \pm 0.5 \mathrm{MeV}$. Of course this is just an attempt to find out, what kind of features could explain the unusual structure in the data. Other less successful attempts tried to explain the energy dependence with nucleon resonances of natural size widths and masses below $\eta^{\prime}$ threshold. Further possibilities could be explored in dynamical coupled channels models with strong background contributions from the $\eta^{\prime} p$ channel, which is, however, not so likely, as eta and etaprime photoproduction have rather small background contributions compared to pion or kaon photoproduction. Nevertheless it is an interesting aspect which deserves further experimental studies with high precision and perhaps additional polarization observables.

\section{Summary and Conclusions}

Here we present a new update of EtaMAID for $\eta$ and $\eta^{\prime}$ photoproduction with four channels, $\eta p, \eta n, \eta^{\prime} p$, and $\eta^{\prime} n$. A large amount of data has been measured during the last decade, mostly from A2MAMI, CBELSA and CLAS. Some of the new polarization observables showed large discrepancies with our previous solutions EtaMAID2001 and EtaMAID2003, and gave therefore a lot of insight in further details of the partial wave analysis. In a new approach, the high-energy regime $W>2.5 \mathrm{GeV}$ was first described with a Regge approach, and the resonance regime from threshold up to $W<2.5 \mathrm{GeV}$ with $21 N^{*}$ resonances for $(\gamma, \eta)$ and $12 N^{*}$ resonances for $\left(\gamma, \eta^{\prime}\right)$. All known $N^{*}$ states listed by PDG have been investigated and, except for only 2 cases, an improvement in our fit was found. Resonances found to be insignificant for our analysis are $N(2040) \frac{3}{2}^{+}$(a one-star state only seen in $J / \Psi$ decays) 
and $N(2220) \frac{9}{2}^{+}$(a four-star high spin state mainly seen in $\pi N$ ). In order to avoid or at least strongly reduce the double counting from Regge plus resonances, we introduced damping factors for $t$-channel exchange contributions.

We obtained very good fits to almost all data, except for some cases, where data from MAMI and CBELSA were in conflict and it did not make sense to use both in the database for our fit. In these cases we decided to use the MAMI data. From all $N^{*}$ resonances that were significantly improving our fits, we found as most significant for $(\gamma, \eta): N(1535) \frac{1}{2}^{-}$, $N(1650) \frac{1}{2}^{-}, N(1895) \frac{1}{2}^{-}, N(1710) \frac{1}{2}^{+}, N(1720) \frac{3}{2}^{+}, N(1520) \frac{3}{2}^{-}, N(1700) \frac{3^{-}}{2}, N(1875) \frac{3}{2}^{-}$, and $N(1860) \frac{5}{2}^{+}$. For $\left(\gamma, \eta^{\prime}\right)$ these are $N(1895) \frac{1}{2}^{-}, N(1880) \frac{1}{2}^{+}, N(1860) \frac{5}{2}^{+}$, and $N(1990) \frac{7^{+}}{2}$.

Generally, in a Breit-Wigner resonance analysis, the resonance parameters are subject to model dependence. This could be rather weak for prominent resonances with widths $\Gamma \lesssim 120 \mathrm{MeV}$, but for broad resonances with widths of several hundred $\mathrm{MeV}$, the model dependence can be very large. Therefore, we plan to perform a detailed resonance analysis with a search of $t$-matrix poles and residues. In an application of the $\mathrm{L}+\mathrm{P}$ method, successfully applied in pion elastic scattering and pion photoproduction, we can expect to reduce the model dependence for the resonance properties considerably.

This work was supported by the Deutsche Forschungsgemeinschaft (SFB 1044).

\section{References}

[1] L. Tiator, Few Body Syst. 59, 21 (2018)

[2] D. Drechsel, O. Hanstein, S. S. Kamalov, and L. Tiator, Nucl. Phys. A 645145 (1999); http://www.kph.uni-mainz.de/MAID/

[3] W. T. Chiang, S. N. Yang, L. Tiator, and D. Drechsel, Nucl. Phys. A 700, 429 (2002)

[4] W. T. Chiang, S. N. Yang, L. Tiator, M. Vanderhaeghen, and D. Drechsel, Phys. Rev. C 68, 045202 (2003)

[5] L. Tiator, M. Gorchtein, V. Kashevarov, K. Nikonov, M. Ostrick, H. Osmanović, M. Hadžimehmedović, R. Omerović, J. Stahov, and A. Švarc, arXiv:1807.04525 [nuclth], submitted to EPJA

[6] M. Tanabashi et al. [Particle Data Group], Phys. Rev. D 98, 030001 (2018)

[7] V. L. Kashevarov, M. Ostrick, and L. Tiator, Phys. Rev. C 96, 035207 (2017)

[8] K. Nikonov, PhD thesis, Mainz 2018

[9] V. L. Kashevarov et al., [A2 Collaboration at MAMI], Phys. Rev. Lett. 118, 212001 (2017)

[10] D. Werthmüller et al., [A2 Collaboration at MAMI], Phys. Rev. C 90, 015205 (2014)

[11] M. Williams et al. [CLAS Collaboration], Phys. Rev. C 80, 045213 (2009)

[12] I. Jaegle et al. [CBELSA/TAPS Collaboration], Eur. Phys. J. A 47, 11 (2011)

[13] V. Krusche and C. Wilkin, Prog. Part. Nucl. Phys. 80, 43 (2015)

[14] A. Švarc et al., Phys. Rev. C 97, 054611 (2018)

[15] A. Švarc et al., arXiv:1807.02759 [nucl-th], submitted to Phys. Rev. C

[16] H. Osmanović, M. Hadžimehmedović, R. Omerović, J. Stahov, V. Kashevarov, K. Nikonov, M. Ostrick, L. Tiator, and A. Švarc, Phys. Rev. C 97, 015207 (2018)

[17] A. V. Anisovich et al., arXiv:1803.06814v1 [nucl-ex]

[18] A. V. Anisovich et al., Phys. Lett. B 772, 247 (2017)

[19] D. Rönchen, M. Döring, and U.-G. Meißner, arXiv:1801.10458v1 [nucl-th]

[20] B. C. Hunt and D. M. Manley, arXiv:1804.06031v1 [nucl-ex] 